\title{
Acoustic impedance of orifices with grazing flow
}

\author{
Diana B ejsenbaeva ${ }^{1}$, Alexandr K omkin $^{1 *}$, Mikhail M ironov ${ }^{1,2}$ and Valeriy M oskal enko ${ }^{1}$ \\ ${ }^{1}$ B auman M oscow State Technical U niversity, Power Engineering Department, 105005, M oscow, \\ Russia \\ ${ }^{2}$ A ndreyev A coustics Institute, Theoretical Department, 117036, M oscow, Russia
}

\begin{abstract}
The paper presents an overview of the results of studies of acoustic impedance of orifices with grazing airflow. Empirical models of orifice impedance, which take into account the grazing flow, and the existing methods of measuring the acoustic impedance of orifices and the experimental setup created for this purpose are analyzed. A simpler way of measuring the acoustic characteristics of an orifice with grazing flow is considered. The scheme of the developed measuring installation and its general appearance are given.
\end{abstract}

\section{Introduction}

Perforated elements are widely used in mufflers for intake and exhaust systems of engines [13]. As a rule, perforated elements are exposed to grazing flow, and it is known that this flow can significantly affect the acoustic impedance of orifices in the perforated elements. Accurate knowledge of the acoustic impedance of the perforation is important for predicting the acoustic performance of the entire muffler.

The acoustic impedance of a perforation without flow is determined by the ratio between the acoustic pressure and the particle velocity inside the perforation, which is described by the concept of an additional mass in an orifice. Rayleigh [1] suggested a model in which air particles oscillate in unison like an incompressible air piston excited by a falling wave when the thickness of the perforation is less than its wavelength. When determining the kinetic energy of an air piston, the concept of end correction length was introduced. Crandall [2] expressed the impedance of the orifice through the wavenumber and length of the Stokes' viscous wave. Sivian [3] derived the formula for the reactive impedance of an orifice from the Raleigh model and also refined the formula for the specific resistance of an orifice by classifying the physical conditions into the Poiseuille and Helmholtz modes. Energy losses from thermal conductivity were assumed to be negligible compared to viscous losses expressed through surface resistance. Ingard [4] derived an orifice resistance formula using the thickness of the viscous boundary layer. It was suggested that the acoustic impedance of the orifice arises from two viscous loss mechanisms: one internal, connected with viscous loss in the orifice itself, and the other external. External loss has been described in terms of a viscous correction of the length of the orifice. Melling [5] included the effect of the interaction of neighboring orifices into the theoretical model. The effect of the interaction of

* Corresponding author: akomkin@mail.ru 
orifices on the impedance was given depending on the distance between the orifices. Sullivan and Crocker [6] measured the impedance of perforated elements in a concentric tube resonator and then modified the theoretical Melling model [5] for orifices with different diameters and thicknesses. All of the above studies on orifice impedance in the absence of constant flow were put into consideration.

A lot of work is devoted to modeling the acoustic impedance of orifices with grazing airflow [7-25]. It should be noted that in this case, strict mathematical modeling of the mechanisms that determine acoustic impedance is significantly more complicated. For this reason, most of the existing impedance models are empirical and, besides, most of them are only for the conditions of the grazing average flow.

The aim of this work is to review the experimental studies carried out in this direction. It consists in the analysis of measurement techniques, comparative evaluation of measurement results taking into account the approximation of the results obtained by analytical expressions, as well as the development of an improved approach to measurements of the acoustic impedance of orifices.

\section{Measurement of orifices acoustic impedance on the sidewall of the duct}

The acoustic resistance of perforated elements as the ratio of the pressure difference between the front and backside of the perforated elements to the particle velocity inside the perforated elements in the form

$$
Z=\frac{p_{f}-p_{r}}{u \prime}
$$

where $Z$ is the acoustic impedance, $p_{f}$ is the sound pressure from the front side of the perforation, $p_{r}$ is the sound pressure from the backside of the perforation and $u^{\prime}$ is the average particle velocity in the orifice of the perforation.

A typical scheme of an experimental setup for measuring the acoustic impedance of a perforation orifice is shown in Figure 1.

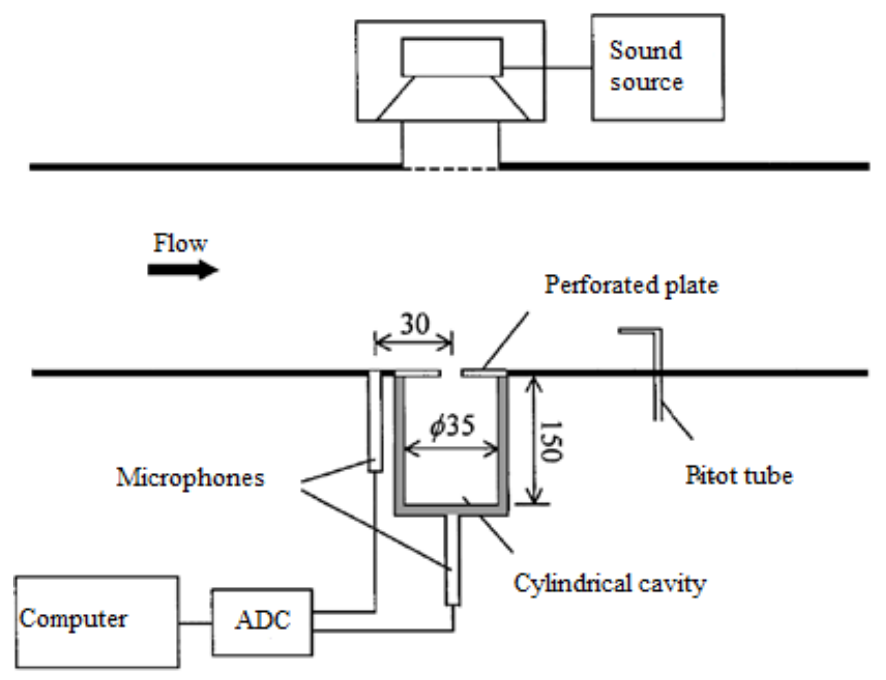

Fig. 1. A typical setup for measuring of the orifice impedance. 
In this setup, the flow velocity is measured with the Pitot tube. The microphone installed behind the face with the inner surface of the duct measures the sound pressure in front of the perforation orifice. The sound pressure measured by a second microphone in a cavity with known cavity sizes can be recalculated to the sound pressure behind the perforation orifice. The value of the oscillating velocity in the orifice [19] can also be obtained by recalculation. As an alternative, a thermoanemometer can be used to directly measure the velocity in the orifice [8]. In addition, these types of measuring setup may differ in the location of the measuring microphones and the sound source.

\section{Empirical formulas for acoustic impedance of orifices}

In the absence of a theory describing the impedance of the orifice in the presence of a grazing flow, the results of measuring this impedance and the subsequent approximation of the experimental results by analytical formulas are of great importance. This approach allows the measurement results to be used in predicting the acoustic characteristics of perforated resonator elements in practical silencer systems. The material in this section is based on a review by Lee and Ih [19].

Garrison [7] proposed an empirical formula of the orifice impedance in the presence of grazing average flow, in which the impedance formula requires a tangential number of the Mach moving flow over the orifice:

$$
Z=(1+1.9 M) R_{0}+j(1-1.65 M) I_{0}
$$

where $R_{0}$ and $I_{0}$ are resistance and reactance respectively in the absence of an average flow. $M$ is the Mach number of the grazing average flow.

Bauer [9] considered both a grazing and a transverse flow for perforation with volumetric elements on its backside and proposed an empirical model. The reactive component of the impedance did not take into account the influence of the flow, and the porosity of the orifices included both the active and reactive components of the impedance, thus taking into account the effect of the interaction of the orifices. He proposed the following empirical formula:

$$
Z_{n}=\left[\left(\frac{\sqrt{8 \mu \rho_{0} \omega}}{\rho_{0} c_{0} \sigma}\right)\left(1+\frac{t}{d}\right)+\frac{0.3 M}{\sigma}+\frac{1.15\left(\frac{\overline{U_{0}}}{c_{0}}\right)}{\sigma}\right]+j\left[\frac{k(t+0.25 d)}{\sigma}\right]
$$

Here $\rho_{0} c_{0}$ is the characteristic impedance of the medium, in which $\rho_{0}$ and $c_{0}$ are the density and velocity of sound, respectively. $Z_{n}$ is the normalized acoustic impedance, which is divided by $\rho_{0} c_{0}, \overline{U_{0}}$ is the transverse flow velocity through the perforation, $\mu$ is the medium viscosity coefficient, $\sigma$ is the perforation porosity, $d$ is the orifice diameter, $\mathrm{t}$ is the orifice thickness, $\omega$ is the circular frequency, and $k$ is the wavenumber.

Sullivan [10] modified his impedance model for the zero flow condition [6] by including the flow effect by reference to the Bauer empirical model [9]. The transverse flow rate was only used for the resistance model, while the reactance, which decreases with increasing flow rate, was accepted as being the same as for the no flow condition:

$$
Z_{n}=\left[\frac{2.57 \overline{U_{0}}}{c_{0}}+j k(t+0.75 d)\right] / \sigma .
$$

Jayaraman and Yam [11] modified the Sullivan impedance model, in which the velocity of the grazing flow was significant only on the resistance model: 


$$
Z_{n}=\left(\frac{0.514 d M}{t \sigma}+j 4.8 \times 10^{-5} f\right) / \sigma
$$

where $f$ is the cyclic frequency.

Rao and Munjal [14] determined acoustic impedance using the impedance tube method and proposed the following empirical model, taking into account all significant parameters such as perforation diameter and thickness, porosity, and grazing average flow rate. The flow effect was only considered in the resistance model.

The normalized acoustic impedance of an orifice in a perforated pipe was empirically set as

$$
Z_{n}=\left[7.337 \cdot 10^{-3}(1+72.23 M)\right] / \sigma+j\left[2.2245 \cdot 10^{-5} \cdot f(1-51 t)(1-204 d)\right] / \sigma \cdot{ }_{(6)}
$$

The permitted input data ranges were: $0.05 \leq M \leq 0.2,0.03 \leq \sigma \leq 0.1, \quad 1 \leq t \leq 3 \mathrm{~mm}$, $1.75 \leq d \leq 7 \mathrm{~mm}$.

Similarly, the results of acoustic impedance measurements of the orifice were approximated by the empirical formulas from Lee and Ih [19]. The actual and imaginary parts of the impedance are functions of perforation porosity, of the orifice diameter and the thickness as well as of the frequency and the Mach number for the grazing flow. To better match the experimental data, the approximating expressions for the real and imaginary parts of the impedance contain five constants each. The advantage of this work is the comparison of the used approximation with the approximations of other authors.

Another approach to approximating experimental data is based on the use of dimensionless parameters. Ronneberger [8] measured the acoustic impedance of perforation with grazing flow as a function of the inverse of the Struhal number, defined as the ratio of the velocity of grazing flow to the product of the orifice radius by the circular frequency.

Later, the so-called friction velocity $u_{\tau}$, was introduced for this purpose. It is described by the expression:

$$
u_{\tau}=\sqrt{\tau_{w} / \rho_{0}}
$$

where $\tau_{w}$ is the shear stress on the duct wall, determined by the average grazing flow velocity $U_{g}$ and the Darcy coefficient of friction $\lambda_{D}$ as follows

$$
\tau_{w}=\rho_{0} U_{g}^{2} \lambda_{D} / 8
$$

The value of $\lambda_{D}$ depends in its turn on Reynolds Re number for the considered duct with the flow. In laminar mode, the Darcy coefficient is independent of roughness and is determined by the Poiseuille formula: $\lambda_{D}=64 /$ Re. In turbulent mode for smooth pipes the Blazyus formula is used: $\lambda_{D}=0,316 / \operatorname{Re}^{1 / 4},\left(10^{4}<\operatorname{Re}<10^{5}\right)$.

Note also that in this case, when describing the impedance of orifices by empirical formulas, the friction velocity $u_{\tau}$ is deformed, resulting in the appearance:

$$
\overline{u_{\tau}}=\frac{u_{\tau}}{(f d)}
$$

This approach seems to go back to Koi and Sarin [11]. In doing so, they suggested that the acoustic impedance of the orifice should be determined empirically through flow resistance and the ratio of end corrections. Flow resistance refers to the change in resistance caused by the flow, which actually means the difference between the measured resistance and the viscosity loss in the orifice. The ratio of end corrections implies the ratio of correction lengths in the presence and absence of grazing flow. 


$$
\begin{aligned}
& R_{f} \frac{c_{0}}{f d}=(5-t / d)\left(9.9 \overline{u_{\tau}}-3.2\right) / 4 \\
& \delta / \delta_{0}=0.92-0.75 \overline{u_{\tau}}+0.11\left(\overline{u_{\tau}}\right)^{2}, 0.2 \leq \overline{u_{\tau}} \leq 3.5 \\
& \delta / \delta_{0}=-0.04, \overline{u_{\tau}}>3.5
\end{aligned}
$$

Here $R_{f}$ denotes the area-averaged resistance to the flow of a single orifice, which can be obtained through porosity with the flow-induced change in resistance of the whole perforated sample, $\delta$ is the corrective length in the presence of an grazing flow, and $\delta_{0}$ is the corrective length in the absence of an grazing flow.

Cummings [15] modified the empirical impedance model of Kooi and Sarin [12] by presenting it in the form:

$$
\begin{gathered}
R_{f} \frac{c_{0}}{f d}=\left(12.52\left(\frac{t}{d}\right)^{-0.32}-2.44\right) \overline{u_{\tau}}-3.2 \\
\delta / \delta_{0}=1, \overline{u_{\tau}} \leq 0.12 \\
\delta / \delta_{0}=(1+0.6 t / d) \exp \left[-\left(\frac{\overline{u_{\tau}} d}{t}-\frac{0.12 d}{t}\right) /(0.25+t / d)\right]-\frac{0.6 t}{d},\left(\overline{u_{\tau}}>0.12\right)
\end{gathered}
$$

Kirby and Cummings [16] later measured the acoustic impedance of the perforation with and without the porous substrate. An empirical impedance model of perforation without a porous substrate was presented as:

$$
\begin{gathered}
R_{f} \frac{c_{0}}{f d}=\left(26.16\left(\frac{t}{d}\right)^{-0.17}-20\right) \overline{u_{\tau}}-4.06 \\
\delta / \delta_{0}=1, \overline{u_{\tau}} \leq 0.12 \\
\delta / \delta_{0}=(1+0.6 t / d) \exp \left[-\left(\frac{\overline{u_{\tau}} d}{t}-\frac{0.18 d}{t}\right) /(1.8+t / d)\right]-\frac{0.6 t}{d},\left(\overline{u_{\tau}}>0.18\right)
\end{gathered}
$$

In the above models considering turbulent effects, the normalized acoustic impedance $R_{n}$ consists of the viscous loss effect $R_{0}$ in the orifice and the flow resistance $R_{f}$ as follows:

$$
R_{n}=R_{f}+R_{0}
$$

The viscous loss is defined as

$$
R_{0}=\frac{\sqrt{8 v \omega}}{c_{0}} \frac{t}{d^{\prime}}
$$

where $v$ is the kinematic viscosity. The normalized acoustic reactance $I_{n}$ can be obtained from an $\delta / \delta_{0}$ end-correction ratio by assuming that the length of the end-correction without flow is approximately $0.85 d$ :

$$
I_{n}=k\left[t-\frac{\delta}{\delta_{0}}(0.85 d)\right]
$$

Dickey and Selamet [18], using a similar approach, obtained simpler approximating formulas for the orifice impedance. So the dimensionless resistance of the orifice is represented as:

$$
\frac{R}{\rho \omega d}=9.57 \overline{u_{\tau}}-0,32, \overline{u_{\tau}} \geq 0.05 .
$$


The imaginary part of the impedance was described by them through a dimensionless equivalent length of the orifice where $l_{e q 1}$ and $l_{e q 2}$ are equivalent lengths of orifices respectively with and without flow. At the same time, this value was presented in the form:

$$
\begin{aligned}
& \overline{l_{e q 1}}=1, \overline{u_{\tau}} \leq 0.03 \\
& \overline{l_{e q 2}}=0.58+0.42 \exp \left[-23.6\left(\overline{u_{\tau}}-0.03\right)\right], \overline{u_{\tau}} \geq 0.03 .
\end{aligned}
$$

As can be seen from the data presented, approximation expressions for impedance orifices obtained by different authors may differ significantly.

The main difference between the presented models of the impedance is that the parameters included in various impedance formulas in earlier models, for example, in $[7,9,10,19]$, are certainly different from the parameters in later models $[12,15,16,18]$. The main ultimate goal of this type of research is to integrate both types into a single format so that the resulting impedance model can easily be used in practical situations, for example, for the exhaust silencing system of internal combustion engines.

Therefore, there are many analytical and empirical impedance models of the orifice. However, the acoustic performance of the orifices they predict, often shows a large discrepancy with the measured results.

\section{Experiment setup}

In this paper, in order to obtain more reliable results for the evaluation of the acoustic impedance of orifices in the presence of grazing flow was proposed a simpler approach to measuring this characteristic and implementing its experimental installation. Studies should take into account such parameters as orifice diameter, orifice thickness, perforation factor, average flow rate and sound frequency.

The scheme of the experimental setup is presented in Figure 2. In the impedance tube 1, the sound field is excited by the sound source 2, which is the speaker. On the other side of the pipe, the sample plate with orifice 3 is placed. On the outer side of the plate is streamlined by grazing airflow 4. The impedance tube contains a system of two condenser microphones (5), which are used to distinguish the amplitudes of sound waves (6) falling on the plate (6) and reflected from it. Knowing these values, we will determine the reflection coefficient $R$, and then the acoustic impedance of the $Z$ plate and the impedance $Z_{0}$ of the orifice itself. In the experimental setup, it is possible to measure the velocity of the grazing flow using a Pitot tube and, on this basis, to obtain the desired dependence of the orifice impedance on the velocity of the grazing flow.

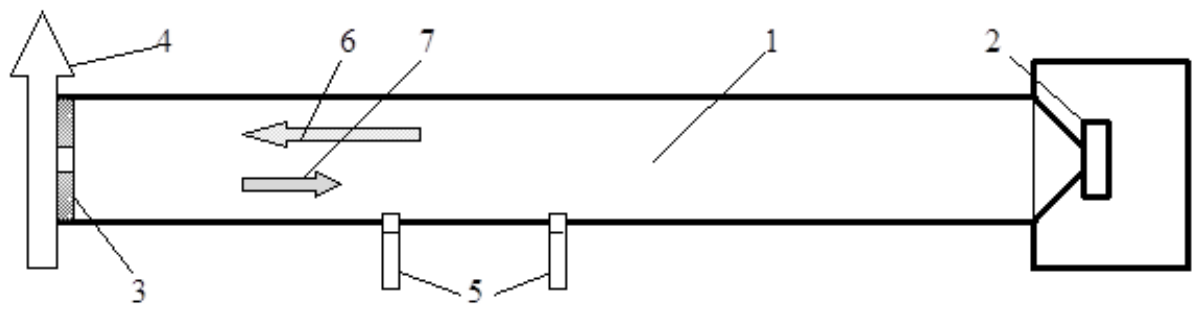

Fig. 2. Experimental setup diagram: 1 impedance tube; 2 sound source (loudspeaker); 3 test plate with an orifice; 5 measuring microphones; 4 grazing airflow; 6, 7 respectively incident and reflected sound waves. 
An experimental setup was created for measurements, shown in Figure 3. It includes an impedance pipe with an internal diameter $\mathrm{d}=99 \mathrm{~mm}$. At one end of the impedance tube, the test plate with an orifice with a diameter of $\mathrm{d} 0$ is installed, and at the other end, it is installed a speaker with a power of $450 \mathrm{~W}$. The input signal for the speaker was generated by a signal generator. A narrowband chirp with $10 \mathrm{~Hz}$ bandwidth was used, which was fed to the speaker through a power amplifier. The amplitude of the chirp varied, so that the sound pressure levels in the impedance tube could vary over a wide range. At the same time, the plate under test can be blown by grazing airflow, which was fed through the slit nozzle from the household vacuum cleaner. The airflow velocity could be adjusted between $5 \mathrm{~m} / \mathrm{s}$ and $50 \mathrm{~m} / \mathrm{s}$. The sound pressure in the impedance tube is measured by a pair of $1 / 4$-inch capacitor microphones. Signals from microphones were then subjected to spectral analysis as they passed through the signal amplifier. Then the received spectra were processed in the computer by the method of transfer functions $[23,24]$ to obtain sound pressure characteristics directly at the partition with an orifice. Simultaneously with acoustic measurements and processing, the Pitot tube [25] measures the vibrational velocity in the septal opening and its surroundings along the longitudinal axis of symmetry.

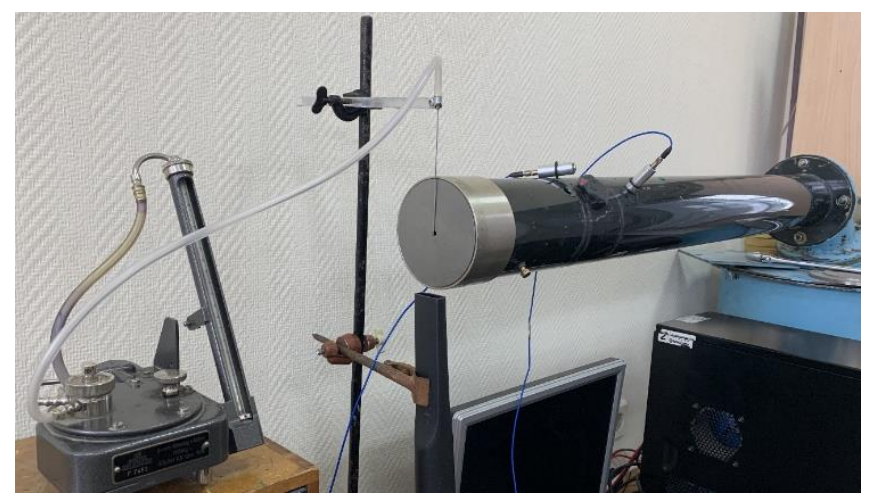

Fig. 3. General view of the experimental setup.

\section{Conclusion}

The review of studies on the evaluation of acoustic impedance with the flow of orifices has allowed us to establish that this problem is very relevant and a large number of works are devoted to it. A large number of empirical formulas describing the acoustic impedance of the orifice obtained from experimental studies are proposed. These formulas do not always lead to the same results and often do not correspond to the results of the theory. This is partly due to measurement errors inherent in the scheme and method of measurement used. This paper considers a simpler measurement scheme that will improve the reliability of the results. An experimental setup was created and debugged to implement this scheme.

\section{References}

1. J. W. Strutt (B aron Rayleigh), The Theory of Sound. (M CM illan, M ichigan, 1896, 2 1896)

2. I. B. Crandall, Theory of V ibrating Systems and Sound, (D. V an N ostrand Company, 1926)

3. L. J. Sivian, J. A coust. Soc. A m. 7 (3), 94 (1935)

4. U. Ingard, J. A coust. Soc. A m. 25 (6), 1037 (1953) 
5. H. M elling, J. Sound Vib. 29 (1), 1 (1973)

6. J. W. Sullivan, M. J. Crocker, J. A coust. Soc. A m. 64 (2), 207 (1978)

7. G. D. Garrison, Pratt and Whitney A ircraft Rep. PW A FR 3299 (1969)

8. D. Ronneberger, J. Sound V ib., 24 (1), 133 (1972).

9. A. B. B auer, J. A ircr. 14 (8), 720-728 (1977)

10. J. W. Sullivan, J. A coust. Soc. A m., 66 (3), 779 (1979)

11. K. Jayaraman, K. Y am, J. A coust. Soc. A m., 69 (2), 390 (1981)

12. J. W. K ooi, S. L. Sarin, A IA A Pap. 81-1998 (1981)

13. A. Goldman, C. H. Chung, J. A coust. Soc. A m. 71 (2), 573 (1982)

14. K. N. Rao, M. L. M unjal, J. Sound Vib. 108 (2), 283 (1986)

15. A . Cummings, A cta A cust. Unit. A cust. 61 (1), 233 (1986)

16. B. Kirby, A. Cummings, J. Sound Vib. 217 (4), 619 (1998)

17. X. Jing, X. Sun, J. W U, K. M eng, A IA A Journal. 39, 1478 (2001)

18. N.S. Dickey, A. Selamet, J. A coust. Soc. A m. 110 (5), 2360 (2001)

19. S-Y. Lee, J-G. Ih, J. A coust. Soc. A m. 114 (1), 98 (2003)

20. K. S. Peat, J-G. Ih, S-H. Lee, J. A coust. Soc. A m. 114 (6), 3076 (2003)

21. G. K ooijmana, A. Hirschberg, J. Golliard, J. Sound V ib. 315 (4), 849 (2008)

22. Y. M eng, B. Xin, X. Jing, X. Sun, H. Bodén, M. Åbom, Semi-empirical impedance model of perforated plate under grazing flow, in proceedings of 25th A IA A/CEAS A eroacoustics Conference, 23 M ay 2019, D elft, The N etherlands (2019)

23. A. I. Komkin, M easur. Techn. 46 (3), 290 (2003)

24. A. I. Komkin, A. I. Bykov, M. A. M ironov, A coust. Phys. 64 (3), 287 (2018)

25. A. Bykov, A. Komkin, V. M oskolenko, Measurements of acoustic flow parameters in the orifice on non-linear regimes, in proceedings of IOP Conf. Series: M aterials Science and Engineering. 589. 012015 (2019) 